\title{
Human Donor Milk: How the Risks to Infants Have Changed
}

Mitchell Goldstein, MD

\section{Introduction}

Breastfeeding is proven to reduce the risk of a wide range of illnesses and conditions. Compared with formula-fed children, Children that receive breastmilk have a reduced risk of ear, skin, stomach, and respiratory infections, diarrhea, sudden infant death syndrome, and necrotizing enterocolitis. In the longer term, breastfed children have a reduced risk of obesity, diabetes, asthma, and childhood leukemia.(1-3) Premature infants benefit even more from human milk. Decreases in cardiovascular disease and hypertension have been seen in ex-premature infants not exposed to cow's milk in the first few weeks of life. (4-7)

For preterm infants born at less than 1,250 grams, the evidence of the benefits of an exclusive human milk diet, devoid of foreign protein (defined as mother's milk and/or pasteurized donor milk plus a human milk-based fortifier), is conclusive. Numerous studies $(3,6,8-10)$ published in major journals examine the difference between feeding premature infants who receive cow milk versus human milk. The studies show a clear and direct relationship between exposure to cow milk-based nutrition and an increased risk of significant morbidities of prematurity.

These studies demonstrate the following benefits for infants receiving an exclusive human diet compared to those exposed to cow milk-based products: (5-7, 9-18)

- Significant reductions in the incidence of necrotizing enterocolitis (NEC)

- Decreased length of hospital stays

- $\quad$ Reduction of the number of total parenteral nutrition (TPN) days

- $\quad$ Reduced feeding intolerance

- Improved weight and length velocity with the proper feeding protocol and the use of a human milk-based fortifier

- $\quad$ Lower mortality

- Reduced incidence of late-onset sepsis

- $\quad$ Reduced incidence of retinopathy of prematurity

- $\quad$ Reduced incidence of bronchopulmonary dysplasia (BPD)

In recent years, the use of donor human milk and human milkderived products has dramatically increased. With a mountain of growing evidence that human milk is best for all babies - and especially critical for premature infants spending time in the Neonatal Intensive Care Unit (NICU) - demand for donor human milk has grown year after year. The Human Milk Banking Association of North America (HMBANA), the largest distributor of pasteurized donor human milk, published in their annual report that the distribution of pasteurized donor milk has increased sevenfold since 2000. HMBANA distributed less than half a million ounces in 2000; In 2019, close to 7.4 million ounces were distributed. (19) Donor human milk is a standard of care in approximately $90 \%$ of the level 3 and 4 NICUs across the country. (20) This is an undoubtedly positive trend, with dozens of studies showing the benefits of keeping premature infants on a diet of strictly human milk and not risking exposure to foreign proteins. This trend has saved countless lives and reduced the cost of care for these vulnerable infants while sending them home to their families even sooner. $(12,13$, 21 ) One studied demonstrated a $\$ 15,750$ savings per day, and 9 fewer days on TPN. $(12,13)$

\section{Incremental costs in these fragile infants can substantially increase the cost of NICU hospitalization.} \author{
costs include: \\ - Surgical NEC $\$ 198,040$ \\ - Medical NEC $\$ 74,004$ \\ - Late onset sepsis $\$ 10,055$ \\ - BPD $\$ 31,565$ \\ - ROP requiring surgery $\$ 35,749$ \\ - PDA $\$ 49,457$
}

Aggregate (of co-morbidities and interventions)

However, one critical component has received very little attention with this growing trend: the safety of donor human milk and human milk-derived products and the milk banks that sell them. Unless the milk is processed under intense scrutiny, human milk is at risk of viral and bacterial contamination. What every parent and/or provider needs to know, especially as donor human milk becomes a more common standard of care, is that at present, there is no uniform, minimum set of safety standards, quality protections, or oversight for these products, regardless of from what milk bank they originate.

\section{"With a mountain of growing evidence that human milk is best for all babies - and especially critical for premature infants spending time in the Neonatal Intensive Care Unit (NICU) - demand for donor human milk has grown year after year."}

We have long studied and worked to eliminate the transmission of viruses, bacteria, and drugs that can be passed through the transfer of human tissue. Newly identified pathogens like SARSCoV-2, coupled with the opioid crisis, recent increases in vaping of nicotine and other substances, and the legalization of marijuana have contributed to increased risks when any human tissue is used anywhere, particularly in the NICU. The effect of these potentially harmful contaminants on breastmilk is difficult to ascertain or quantify because of an inability to gauge the level of exposure transmitted through milk. What we do know, however, is that exposure to even minute doses of many of these substances can lead to severe and life-long negative consequences for ill and fragile premature newborns. $(22,23)$ Moreover, because of the long-term consequences of exposure to these contaminants, evidence of exposure may not even present until long after birth or hospital discharge. Claims of safety and a paucity of traceable pathology notwithstanding, there is no credible way of assessing human donor milk because of the inconsistency inherent in these exposures. The lack of consistent regulation and oversight of this vital medical tissue, human milk, places infants at unnecessary risk. 


\section{What Are the Risks?}

Milk is both a tissue and a biologic fluid derived from a human, like blood and plasma. Despite the best technology available and a mature understanding of the risks inherent in blood donation practices, clinicians in the 1980s found themselves deficient in recognizing the harbinger of the encroaching Acquired Immunodeficiency Syndrome (AIDS) epidemic. Technology had progressed to the point where threats to the blood supply, including hepatitis and bacterial infection, could be reliably identified and mitigated. Intravenous (IV) drug users were discouraged from giving blood because of these risks. Blood shelf stability had been established, and a uniform reporting mechanism had been set in place to guard against unexpected outcomes. However, in the end, all of these practices were insufficient to deal with Human Immunodeficiency Virus (HIV) because the virus responsible for the challenge was not a previously identified pathogen and was not on the test panel. At-risk behaviors, defined conditions, and factors responsible for the disease's continued spread were yet to be discovered. An entire generation of people living with hemophilia, dependent on plasma from multiple donors, were practically wiped out by screening practices that were insufficient to recognize the risk. (24)

Moreover, victims of trauma, those with chronic anemia, and even pregnancy complications were exposed to tainted blood. By the time the risks were known, it was too late. Millions of people worldwide were ultimately affected, and HIV continues to represent a challenge to communities worldwide.

Nevertheless, there was a yet crueler truth to HIV infection, one we never saw coming. Vertical transmission of HIV infection was not only possible through direct transmission of the virus through pregnancy, but also through breastmilk. Between one-third and one-half of infants born to an HIV-positive mother subsequently went on to develop HIV infection and full-blown (AIDS). $(25,26)$ The tragedy was not fully elucidated until years after identifying the initial risk groups. Without the devoted efforts of a mother affected by this calamity and the concerted efforts of her public supporters, AIDS may have been remained relegated to its assigned position as a disease of moral failure as opposed to a severe health concern for all of us, including breastfeeding infants. (27)

\section{"Nevertheless, there was a yet crueler truth to HIV infection, one we never saw coming. Vertical transmission of HIV infection was not only possible through direct transmission of the virus through pregnancy, but also through breastmilk."}

We learned many lessons from the HIV epidemic of the 1980s, including that enhanced vigilance must be enforced to recognize ongoing threats proactively. We must learn from this. When considering human donor milk and human donor milk-derived products, we must adopt a more vigilant approach, particularly when milk is delivered to preterm and medically fragile infants in the NICU. Many of these threats can be mitigated by pasteurization and other processes, assuming they are conducted appropriately. However, other contaminants cannot be resolved by pasteurization alone. Given the current trend towards increased demand for human donor milk and the emergence of new milk banks entering the field, we must act now to mitigate the risk.
These threats include:

- Bacterial contamination

- Viral transmission

- Nicotine, marijuana, homeopathic remedies, recreational drugs, and other substances that mothers may not realize pass readily through the breastmilk

- $\quad$ Over-the-counter medications (e.g., Sudafed), opioids, and other prescription pain medications for c-section, dental work, chronic pain, or depression

\section{- $\quad$ Breastmilk from unqualified donors}

- Milk from other sources, including bovine, ovine, soy, and oat milk

By way of example, we know that infants' exposure to nicotine results in myriad issues, including liver and lung damage, the potential of an increased susceptibility to diabetes owing to a reduction in pancreatic beta cell production, and, most notably, significant differences in infant sleep-wake cycle. Extensive exposure to nicotine in utero has been associated with withdrawal. (28) These effects may be exacerbated in the preterm infant. (28-32) What is more, approximately $85 \%$ of women who quit smoking during pregnancy resume smoking following the delivery of their babies, often within the first 2-8 weeks postpartum. (33) Milk banks that test incoming milk find that nicotine and its metabolites are the most common contaminant in donated human milk. It is important to note that the infants in these studies were term infants. Because of the small population and ethics involved, randomized control trials of preterm infants' exposure to nicotine are not available. However, several studies focusing on risk identification and conventional wisdom dictate that any effects on a term infant may be magnified in a smaller preterm infant. $(32,34,35)$

Cannabis exposure is increasing, as many states legalize marijuana for both medicinal and recreational use. Despite laws proscribing harsh penalties for the furnishing of any cannabis to minors, even in breastmilk (36), the consequence of de-emphasizing the risk in the adult population heightens the concern for newborns. THC, a principal constituent of marijuana, was measurable in most breast milk samples up to 6 days after maternal marijuana use. $(37,38)$ The enhanced potency of marijuana is concerning since the only study on long term neurodevelopmental outcomes was conducted when THC concentrations in marijuana were estimated to be one-third of today's levels. (37) As THC enters the bloodstream one hour after use and persists for up to six days, it may not be possible to use cannabis and avoid infant exposure. There is building evidence that this exposure may result in cognitive, social, and motor defects. (36-38)

\section{"Despite laws proscribing harsh penalties for the furnishing of any cannabis to minors, even in breastmilk (36), the consequence of de-emphasizing the risk in the adult population heightens the concern for newborns."}

\section{Why Are the Risks Greater Today Than Ten Years Ago?}

Human milk is medicine for sick and premature newborns. Be- 
yond the apparent nutritional benefit, a humoral component includes substances that provide a level of immunoprotection not attainable through other sources. It provides health benefits for all newborn infants but is of critical importance for these high-risk infants. Human milk provides significant mitigation of the excess risk for those at risk of growth failure and developmental delay.

The increased use of donor human milk and donor human milkderived products has saved countless lives and improved NICU care around the country and the world. Statistically significant increases in weight, length, and head circumference growth have been noted. (Huston 2018) Decreased risk of necrotizing enterocolitis (NEC), growth failure, and infection have been documented extensively. Furthermore, because of the increased use of donor human milk, there has been significant growth in the number of human milk banks that collect, process, and sell donor human milk - both for- and not-for-profits. It has become increasingly important to recognize the increase in risk associated with this growth. HMBANA, for example, has nearly doubled the number of milk banks they operate on the continent. In that same period, we have seen several for-profits and other commercial entities enter the scene. Over $30 \%$ of hospitals with advanced services used donor milk in the NICU in 2013; this percentage has increased dramatically since that time, as evidenced by the increase in the demand for donor breast milk and the proliferation of human milk banks. By 2017, the number of level 3 and 4 NICU's with access to donor human milk was $88 \%$. $(39,40)$

Due to the increased demand in the market and a steady stream of new organizations entering the market to meet that demand, it is critically important to not only have an understanding of the types of practices that are in place but to have minimum safety standards for managing the risks and reporting deviations from accepted practice that could adversely affect the milk supply. Furthermore, if issues do arise, it is especially critical to track and trace the source of the issue. $(5,10)$

"Due to the increased demand in the market and a steady stream of new organizations entering the market to meet that demand, it is critically important to not only have an understanding of the types of practices that are in place but to have minimum safety standards for managing the risks and reporting deviations from accepted practice that could adversely affect the milk supply."

\section{How Do We Mitigate the Risk?}

Few if any of the milk banks that sell human donor milk have governmental regulations outside of registering with the US Food and Drug Administration (FDA) as a food manufacturer. Some milk banks have voluntary, self-enforced guidelines. Most human milk banks rely on self-generated screening, production, safety, and quality guidelines that are neither publicly nor independently audited or enforced. Although some of these banks operate as not for profit, this designation does not mitigate safety and truth in marketing. Further, this laissez-faire honor system does not recognize the inherent risk of collecting, processing, and distribut- ing a human biologic fluid, which is why all other biologics are regulated by the government. The only exceptions are milk banks operating in California, Maryland, and New York, where the milk banks must obtain a tissue bank license. Still, these tissue bank licenses do not even begin to address what is necessary to regulate and administer the full scope of breast milk supplementation. This requirement may be why the American Academy of Pediatrics stated that "federal or state guidelines are needed regarding the preparation, handling, and transfer of human milk as well as the operation of donor human milk banks...". (8) These guidelines may be insufficient. Where safety is parament, strict regulations and policies not dissimilar to the blood and plasma industry must be put into place.

For products derived from human milk that go beyond strictly pasteurized donor human milk, regulations vary, with fortifiers more strictly regulated by the FDA as an exempt infant formula. However, even here, improvement in the guidance and recognition of a uniform reporting requirement similar to the plasma industry would enhance consumer protection, and ensure as a safer product for the most vulnerable babies.

It should be noted that the difference between registering as a food manufacturer and being regulated as exempt infant formula is significant. A different branch of the FDA regulates food manufacturers. Regulations are mainly concerned with outward-facing risks inherent to the manufacturing process. Because food product variation is accepted, precise guidance dosing, treatment, and spoilage reporting occur at a different level of significance. Audits, inspections, and adverse event reporting do not have the same level of significance or consequence if they take place at all. The vast majority of human milk banks are regulated as ordinary food products and thus not subject to rigorous or frequent inspection or oversight. (41)

Breastmilk and its derivatives are inherently different from the typical food item or even infant formula. For example, the current guidance does not require testing the donor milk for the viruses, bacteria, drugs, and adulterants outlined above. The veracity of donor self-reporting is critical to the success of this system. At best, in some cases, non-profit milk banks are "self-regulated," with voluntary guidelines but no uniformity or requirements for testing, safety, and quality protocols beyond pasteurization. Further, there is neither a standardized metric for safety nor post hoc analysis required should a problem with the accepted local protocol arise.

Do we know if there have been any infants negatively impacted? Harm cannot be monitored effectively under the current system. What is more, there is no absolute requirement to report adverse events. Individualized sample quality control is non-existent. Randomized testing standard in the food manufacturing industry precludes the best practice to test every batch of donor milk before and after it has been pasteurized, processed, and packaged.

Screening for breaches or variations in practices is dependent on the prevalence of the breach or variation. If one percent of the samples are affected, a screening process checking one out of 100 samples would only be predicted to find the deviation after screening 100 intervals of 100 or after 10,000 samples had passed through the manufacturing process. Significant risk cannot be avoided when significant components of the manufacturing process involving the production, expression, and collection of breastmilk are out of the manufacturer's direct control. (11)

A more rigorous testing system is required. Screening may be appropriate for food items with generally accepted safety profiles and where these items are destined for adult consumption. However, where variations are less well tolerated and potential risks greater, screening protocols must give way to enhanced testing designed 
to accommodate the individualized risk and need for batch identification and recall if indicated, particularly when that product is being fed to premature and medically fragile infants. $(7,8)$

\section{How Do These Risks Impact the Babies?}

In November 2019, three premature infants died due to bacterial contamination of the equipment used to measure and mix donor milk at Geisinger Medical Center in Danville, PA. (42) The scrutiny involved in the maintenance, preparation, and quality assurance was insufficient to prevent excess morbidity and mortality. Proactive anticipation of the next deviation and progressive quality improvement of existing processes are crucial in preventing system failures and their devastating effects on our most at-risk patients.

Evidence also points to both short and long-term health issues of preemies exposed to many exogenous substances with no place in breastmilk. Neonatal Abstinence Syndrome (NAS) has increased dramatically and continues to impact healthcare. The long-term implications of exposure to nicotine, cannabinoid, and other newer substances on neurodevelopment, respiratory conditions, and growth parameters cannot be fathomed. Screening for these practices on a haphazard basis fails to acknowledge the risk. The responsible approach to acknowledging these practices is enhanced testing of threats, both known and anticipated, with efforts dedicated to risk reduction.

"Given the extremely fragile nature of the very low birth weight premature infants, all organizations, for or not for profit, who manufacture and distribute or sell human milk products must join in developing a standard of care methodology for the expression, storage, testing, and proper
safety regulations of this vital resource."

\section{Conclusion:}

The status quo of self-regulated, voluntary guidelines in human donor milk is antiquated and insufficient, given the growth of the use of human donor milk in the NICU. The risk of bacterial and viral infection, coupled with continually changing pathogens and adulterants, must not be discounted. Safety measures should not be interpreted as a monopoly or "competitive advantage" they must compel a deeper level of understanding and collaboration, a responsibility that we must all share. It can no longer be acceptable for a doctor to reach for a human milk product that does not meet these rigorous, yet common sense, safety standards simply because it is what is available. As testing equipment for these potential concerns is widely available, no entity should be granted a pass on compliance. The lack of strong governance relating to the use of best available guidance on safe practices does not excuse a lack of vigilance.

Given the extremely fragile nature of the very low birth weight premature infants, all organizations, for or not for profit, who manufacture and distribute or sell human milk products must join in developing a standard of care methodology for the expression, storage, testing, and proper safety regulations of this vital resource. Although regulations are in place for regulating food products, medications, biologics, and the blood and plasma industry, human milk and its derivatives are clearly distinct from other classes. Albeit a food product, human milk is clearly a biologic and medication as well. The risks inherent in these products are not dissimilar to those in the blood and plasma industry. At this point, too much time has gone by, and too many lives have been placed at risk for this issue to be relegated to an area that is betwixt and between. There is no excuse for the lack of strong guidance and regulatory enforcement. The government must oversee this industry.

\section{References:}

1. Amitay EL, Keinan-Boker L. Breastfeeding and Childhood Leukemia Incidence: A Meta-analysis and Systematic Review. JAMA Pediatr. 2015;169(6):e151025. Epub 2015/06/02. doi: 10.1001/jamapediatrics.2015.1025. PubMed PMID: 26030516.

2. Halken S. Prevention of allergic disease in childhood: clinical and epidemiological aspects of primary and secondary allergy prevention. Pediatr Allergy Immunol. 2004;15 Suppl 16:4-5, 9-32. Epub 2004/05/06. doi: 10.1111/j.13993038.2004.0148b.x. PubMed PMID: 15125698.

3. Binns C, Lee M, Low WY. The Long-Term Public Health Benefits of Breastfeeding. Asia Pac J Public Health. 2016;28(1):714. Epub 2016/01/23. doi: 10.1177/1010539515624964. PubMed PMID: 26792873.

4. Hair AB, Peluso AM, Hawthorne KM, Perez J, Smith DP, Khan JY, et al. Beyond Necrotizing Enterocolitis Prevention: Improving Outcomes with an Exclusive Human Milk-Based Diet. Breastfeed Med. 2016;11(2):70-4. Epub 2016/01/21. doi: 10.1089/bfm.2015.0134. PubMed PMID: 26789484; PubMed Central PMCID: PMCPMC4782036.

5. Huston RK, Markell AM, McCulley EA, Gardiner SK, Sweeney SL. Improving Growth for Infants $</=1250$ Grams Receiving an Exclusive Human Milk Diet. Nutr Clin Pract. 2018;33(5):671-8. Epub 2018/02/17. doi: 10.1002/ ncp.10054. PubMed PMID: 29451716.

6. Jacobi-Polishook T, Collins CT, Sullivan TR, Simmer K, Gillman MW, Gibson RA, et al. Human milk intake in preterm infants and neurodevelopment at 18 months corrected age. Pediatr Res. 2016;80(4):486-92. Epub 2016/06/30. doi: 10.1038/pr.2016.114. PubMed PMID: 27356082.

7. Mizuno K, Shimizu T, Ida S, Ito S, Inokuchi M, Ohura T, et al. Policy statement of enteral nutrition for preterm and very low birthweight infants. Pediatr Int. 2020;62(2):124-7. Epub 2020/02/07. doi: 10.1111/ped.14067. PubMed PMID: 32026585; PubMed Central PMCID: PMCPMC7065204.

8. Committee On N, Section On B, Committee On F, Newborn. Donor Human Milk for the High-Risk Infant: Preparation, Safety, and Usage Options in the United States. Pediatrics. 2017;139(1). Epub 2016/12/21. doi: 10.1542/peds.20163440. PubMed PMID: 27994111.

9. Cristofalo EA, Schanler RJ, Blanco CL, Sullivan S, Trawoeger R, Kiechl-Kohlendorfer $U$, et al. Randomized trial of exclusive human milk versus preterm formula diets in extremely premature infants. J Pediatr. 2013;163(6):15925 e1. Epub 2013/08/24. doi: 10.1016/j.jpeds.2013.07.011. PubMed PMID: 23968744.

10. Huston $R$, Lee $M$, Rider E, Stawarz M, Hedstrom D, Pence $M$, et al. Early fortification of enteral feedings for infants $<1250$ grams birth weight receiving a human milk diet including human milk based fortifier. J Neonatal Perinatal Med. 2020;13(2):215-21. Epub 2019/11/11. doi: 10.3233/ NPM-190300. PubMed PMID: 31707377; PubMed Central PMCID: PMCPMC7369034.

11. Fischer HR, Duncan SD. The business case for quality improvement. J Perinatol. 2020;40(6):972-9. Epub 2020/04/02. doi: 10.1038/s41372-020-0660-y. PubMed PMID: 32231258.

12. Ganapathy V, Hay JW, Kim JH. Costs of necrotizing enterocolitis and cost-effectiveness of exclusively human milkbased products in feeding extremely premature infants. Breastfeed Med. 2012;7(1):29-37. Epub 2011/07/02. doi: 
10.1089/bfm.2011.0002. PubMed PMID: 21718117.

13. Ganapathy V, Hay JW, Kim JH, Lee ML, Rechtman DJ. Long term healthcare costs of infants who survived neonatal necrotizing enterocolitis: a retrospective longitudinal study among infants enrolled in Texas Medicaid. BMC Pediatr. 2013;13:127. Epub 2013/08/22. doi: 10.1186/1471-2431-13127. PubMed PMID: 23962093; PubMed Central PMCID: PMCPMC3765805.

14. Hair AB, Hawthorne KM, Chetta KE, Abrams SA. Human milk feeding supports adequate growth in infants $</=1250$ grams birth weight. BMC Res Notes. 2013;6:459. Epub 2013/11/14. doi: 10.1186/1756-0500-6-459. PubMed PMID: 24220185; PubMed Central PMCID: PMCPMC3879715.

15. Johnson TJ, Patel AL, Bigger HR, Engstrom JL, Meier PP. Cost savings of human milk as a strategy to reduce the incidence of necrotizing enterocolitis in very low birth weight infants. Neonatology. 2015;107(4):271-6. Epub 2015/03/15. doi: 10.1159/000370058. PubMed PMID: 25765818; PubMed Central PMCID: PMCPMC4458214.

16. Maffei $D$, Schanler RJ. Human milk is the feeding strategy to prevent necrotizing enterocolitis! Semin Perinatol. 2017;41(1):36-40. Epub 2016/11/12. doi: 10.1053/j.semperi.2016.09.016. PubMed PMID: 27836421

17. Parker LA, Krueger C, Sullivan S, Kelechi T, Mueller M. Effect of breast milk on hospital costs and length of stay among very low-birth-weight infants in the NICU. Adv Neonatal Care. 2012;12(4):254-9. Epub 2012/08/07. doi: 10.1097/ ANC.0b013e318260921a. PubMed PMID: 22864006.

18. Perrin MT, Belfort MB, Hagadorn JI, McGrath JM, Taylor SN, Tosi LM, et al. The Nutritional Composition and Energy Content of Donor Human Milk: A Systematic Review. Adv Nutr. 2020;11(4):960-70. Epub 2020/03/03. doi: 10.1093/advances/nmaa014. PubMed PMID: 32119744; PubMed Central PMCID: PMCPMC7360450.

19. HMBANA; Donor Human Milk Distribution Increases By Nearly 1 Million Ounces 2020. Available from: https://www. hmbana.org/news/donor-human-milk-increases-by-nearly1-million-ounces.html.

20. Parker MG, Burnham LA, Kerr S, Belfort MB, Perrin M, Corwin $M$, et al. Prevalence and predictors of donor milk programs among U.S. advanced neonatal care facilities. J Perinatol. 2020;40(4):672-80. Epub 2020/02/28. doi: 10.1038/ s41372-020-0620-6. PubMed PMID: 32103161.

21. Assad M, Elliott MJ, Abraham JH. Decreased cost and improved feeding tolerance in VLBW infants fed an exclusive human milk diet. J Perinatol. 2016;36(3):216-20. Epub 2015/11/13. doi: 10.1038/jp.2015.168. PubMed PMID: 26562370.

22. Chavez-Almazan LA, Saldarriaga-Norena HA, Diaz-Gonzalez L, Garibo-Ruiz D, Waliszewski SM. Dietary habits associated with the presence of organochlorine pesticides in human milk. J Environ Sci Health B. 2020;55(8):756-66. Epub 2020/07/15. doi: 10.1080/03601234.2020.1783169. PubMed PMID: 32663053.

23. Peters MD, McArthur A, Munn Z. Safe management of expressed breast milk: A systematic review. Women Birth. 2016;29(6):473-81. Epub 2016/06/20. doi: 10.1016/j.wombi.2016.05.007. PubMed PMID: 27318564.

24. Elizabeth Glaser Pediatric AIDS Foundation. Elizabeth's Story 2020. Available from: https://www.pedaids.org/about/ elizabeths-storyl.

25. Davis NL, Corbett A, Kaullen J, Nelson JAE, Chasela CS, Sichali D, et al. Antiretroviral Drug Concentrations in Breastmilk, Maternal HIV Viral Load, and HIV Transmission to the Infant: Results From the BAN Study. J Acquir Immune Defic Syndr. 2019;80(4):467-73. Epub 2018/12/21. doi: 10.1097/ QAI.0000000000001941. PubMed PMID: 30570527; PubMed Central PMCID: PMCPMC6391211.
26. Ruff AJ. Breastmilk, breastfeeding, and transmission of viruses to the neonate. Semin Perinatol. 1994;18(6):510-6. Epub 1994/12/01. PubMed PMID: 7701353.

27. Glaser E, Palmer L. In the Absence of Angels. 1st ed. New York: G. P. Putnam's Sons; 1991. 319 p.

28. Vagnarelli F, Amarri S, Scaravelli G, Pellegrini M, GarciaAlgar O, Pichini S. TDM grand rounds: neonatal nicotine withdrawal syndrome in an infant prenatally and postnatally exposed to heavy cigarette smoke. Ther Drug Monit. 2006;28(5):585-8. Epub 2006/10/14. doi: 10.1097/01. ftd.0000245391.56176.ad. PubMed PMID: 17038869.

29. Garcia-Algar O, Puig C, Mendez C, Vall O, Pacifici R, Pichini S. Neonatal nicotine withdrawal syndrome. J Epidemiol Community Health. 2001;55(9):687-8. Epub 2001/09/11. doi: 10.1136/jech.55.9.687b. PubMed PMID: 11548709; PubMed Central PMCID: PMCPMC1731974.

30. Garcia-Algar O, Puig $C$, Vall O, Pacifici R, Pichini S. Effects of maternal smoking during pregnancy on newborn neurobehavior: neonatal nicotine withdrawal syndrome. Pediatrics. 2004;113(3 Pt 1):623-4; author reply -4. Epub 2004/03/03. doi: 10.1542/peds.113.3.623-a. PubMed PMID: 14993560.

31. Primo CC, Ruela PB, Brotto LD, Garcia TR, Lima Ede F. Effects of maternal nicotine on breastfeeding infants. Rev Paul Pediatr. 2013;31(3):392-7. Epub 2013/10/22. doi: 10.1590/ S0103-05822013000300018. PubMed PMID: 24142324; PubMed Central PMCID: PMCPMC4182966.

32. Tong VT, England LJ, Rockhill KM, D'Angelo DV. Risks of Preterm Delivery and Small for Gestational Age Infants: Effects of Nondaily and Low-Intensity Daily Smoking During Pregnancy. Paediatr Perinat Epidemiol. 2017;31(2):144-8. Epub 2017/02/10. doi: 10.1111/ppe.12343. PubMed PMID: 28181676; PubMed Central PMCID: PMCPMC6368675.

33. Phillips RM, Merritt TA, Goldstein MR, Deming DD, Slater LE, Angeles DM. Prevention of postpartum smoking relapse in mothers of infants in the neonatal intensive care unit. J Perinatol. 2012;32(5):374-80. Epub 2011/08/13. doi: 10.1038/jp.2011.106. PubMed PMID: 21836549; PubMed Central PMCID: PMCPMC3343345.

34. Jaddoe VW, Troe EJ, Hofman A, Mackenbach JP, Moll HA, Steegers EA, et al. Active and passive maternal smoking during pregnancy and the risks of low birthweight and preterm birth: the Generation R Study. Paediatr Perinat Epidemiol. 2008;22(2):162-71. Epub 2008/02/27. doi: 10.1111/j.13653016.2007.00916.x. PubMed PMID: 18298691.

35. Kyrklund-Blomberg NB, Cnattingius S. Preterm birth and maternal smoking: risks related to gestational age and onset of delivery. Am J Obstet Gynecol. 1998;179(4):1051-5. Epub 1998/10/28. doi: 10.1016/s0002-9378(98)70214-5. PubMed PMID: 9790397.

36. UNIFORM CONTROLLED SUBSTANCES ACT [11000 11651] 11361 (2017).

37. Bertrand KA, Hanan NJ, Honerkamp-Smith G, Best BM, Chambers CD. Marijuana Use by Breastfeeding Mothers and Cannabinoid Concentrations in Breast Milk. Pediatrics. 2018;142(3). Epub 2018/08/29. doi: 10.1542/peds.20181076. PubMed PMID: 30150212; PubMed Central PMCID: PMCPMC6317767.

38. Davis E, Lee T, Weber JT, Bugden S. Cannabis use in pregnancy and breastfeeding: The pharmacist's role. Can Pharm J (Ott). 2020;153(2):95-100. Epub 2020/03/25. doi: 10.1177/1715163519893395. PubMed PMID: 32206154; PubMed Central PMCID: PMCPMC7079319.

39. Parker MG, Burnham LA, Kerr SM, Belfort MB, Perrin MT, Corwin MJ, et al. National Prevalence of Donor Milk Use Among Level 1 Nurseries. Hosp Pediatr. 2020. Epub 2020/11/05. doi: 10.1542/hpeds.2020-001396. PubMed PMID: 33144332.

40. Perrine CG, Scanlon KS. Prevalence of use of human 
milk in US advanced care neonatal units. Pediatrics. 2013;131(6):1066-71. Epub 2013/05/15. doi: 10.1542/ peds.2012-3823. PubMed PMID: 23669517; PubMed Central PMCID: PMCPMC4535053.

41. Updegrove KH. Donor human milk banking: growth, challenges, and the role of HMBANA. Breastfeed Med. 2013;8(5):4357. Epub 2013/10/12. doi: 10.1089/bfm.2013.0079. PubMed PMID: 24112059.

42. Ortiz A. Deaths of 3 Infants Traced to Contaminated Equipment, Hospital Says. New York Times [Internet]. 2019 Nov. 8, 2019. Available from: https://www.nytimes. com/2019/11/08/us/pennsy/vania-infant-deaths-breastmilk.htm/\#: : text=Geisinger\%20Medical\%20Center\%20 in $\% 20$ Danville,cause $\% 20$ of\%20deadly $\% 20$ bacterial\%20 infections. \&text=The $\% 20$ infants $\% 20$ at $\% 20$ the $\% 20$ Geisinger,some $\% 20$ started $\% 20$ to $\% 20$ get $\% 20$ ill.

Disclosure: The author has no disclosures.

NT

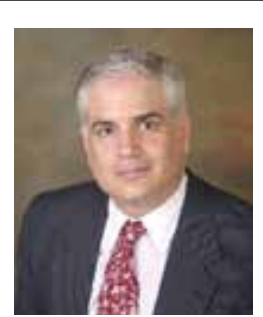

Corresponding Author

Mitchell Goldstein, MD

Professor of Pediatrics

Loma Linda University School of Medicine

Division of Neonatology

Department of Pediatrics

mgoldstein@llu.edu

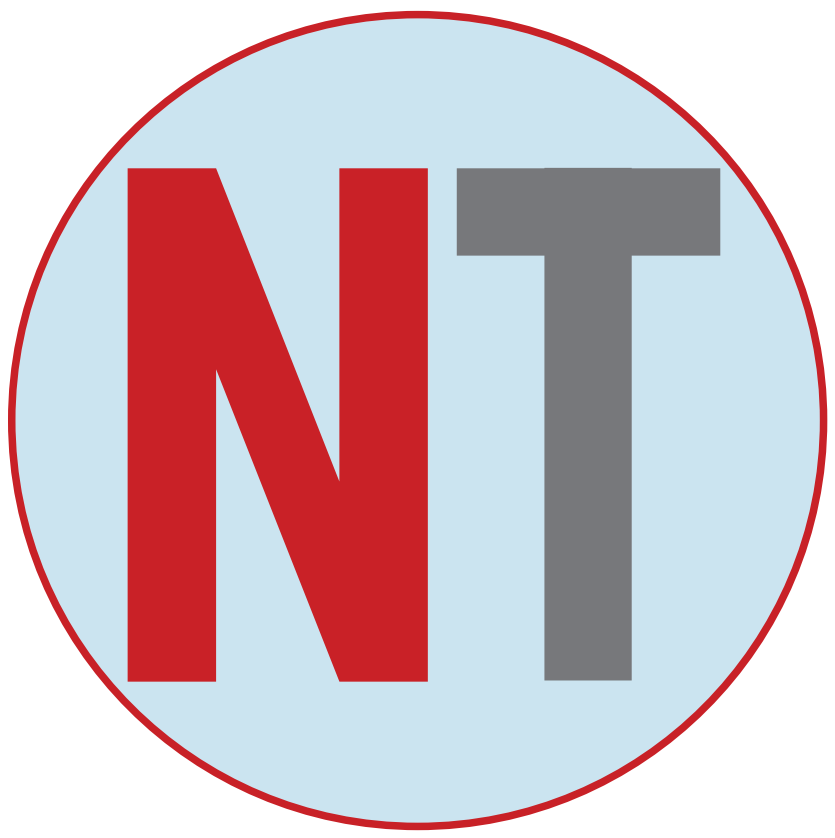

SHARED DECISION-MAKING PROTECTS MOTHERS + INFANTS DURING COVID-19

KEEPING MOTHERS + INFANTS TOGETHER

Means balancing...

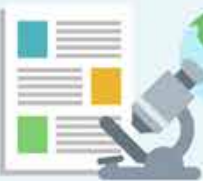

\section{EVIDENCE}

We encourage families and clinicians to remain diligent in learning up-to-date evidence.

\section{PARTNERSHIP}

SHARED DECISION-MAKING

What is the best for this unique dyad?

\section{S EEK PARTICIPATION HELP EXPLORE OPTIONS A SSESS PREFERENCES REACHADECISION \\ EVALUATE THE DECISION}

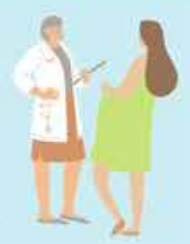

\section{TRAUMA-INFORMED}

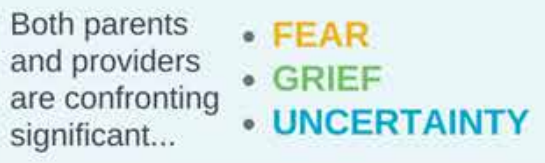

\section{LONGITUDINAL DATA}

We need to understand more about outcomes for mothers and infants exposed to COVID-19, with special attention to:

$$
\text { - MENTAL HEALTH }
$$

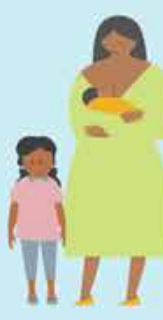

NEW DATA EMERGE DAILY.

NANN AND NPA ENCOURAGE PERINATAL CARE PROVIDERS TO ENGAGE IN CANDID CONVERSATIONS WITH PREGNANT PARENTS PRIOR TO DELIVERY REGARDING RISKS, BENEFITS, LIMITATIONS, AND REALISTIC EXPECTATIONS.

Partnering for patient-centered care when it matters most.

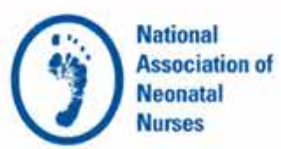

nann.org

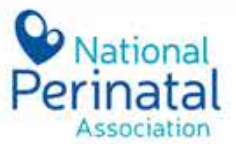

nationalperinatal.org 